\title{
ACCRETION DISK TEMPERATURES OF QSOs: CONSTRAINTS FROM THE EMISSION LINES
}

\author{
E. W. Bonning ${ }^{1,2}$, G. A. Shields ${ }^{3}$, A. C. Stevens ${ }^{3}$, and S. Salviander ${ }^{3,4}$ \\ ${ }^{1}$ Quest University Canada, 3200 University Boulevard, Squamish, BC V8B 0N8, Canada; erin.bonning@questu.ca \\ 2 Yale Center for Astronomy and Astrophysics, Yale University, P.O. Box 208121, New Haven, CT 06511, USA \\ ${ }^{3}$ Department of Astronomy, University of Texas, Austin, TX 78712, USA; shields@ astro.as.utexas.edu, \\ alyx.stevens@mail.utexas.edu, triples@astro.as.utexas.edu \\ ${ }^{4}$ Department of Physics, Southwestern University, Georgetown, TX 78626, USA \\ Received 2012 July 31; accepted 2013 April 13; published 2013 May 21
}

\begin{abstract}
We compare QSO emission-line spectra to predictions based on theoretical ionizing continua of accretion disks. The observed line intensities do not show the expected trend of higher ionization with theoretical accretion disk temperature as predicted from the black hole mass and accretion rate. Consistent with earlier studies, this suggests that the inner disk does not reach temperatures as high as expected from standard disk theory. Modified radial temperature profiles, taking account of winds or advection in the inner disk, achieve better agreement with observation. The emission lines of radio-detected and radio-undetected sources show different trends as a function of the theoretically predicted disk temperature.
\end{abstract}

Key words: black hole physics - galaxies: active - quasars: general

Online-only material: color figures

\section{INTRODUCTION}

The idea of accretion disks around supermassive black holes as the power source of active galactic nuclei (AGN) is widely accepted. Physical plausibility, the lack of successful alternatives, and the frequent presence of bipolar jets support this picture. Qualitatively, the blue-ultraviolet "Big Blue Bump" in the spectral energy distribution (SED) of QSOs resembles the expected thermal emission from the disk atmosphere (Shields 1978; Malkan 1983). Models achieve some success in fitting the SED of individual QSOs (e.g., Hubeny et al. 2001). However, the simple disk model has known shortcomings in accounting for the soft X-ray continuum, optical polarization, variability (e.g., Koratkar \& Blaes 1999). Further difficulties include (1) the "ionization problem" involving a deficit in the number of hard ionizing photons in the extrapolation of observed UV continuum slopes (e.g., Netzer 1985; Korista et al. 1997), (2) the "size problem" resulting from microlensing measurements giving optical continuum sizes larger that expected from disk theory (Pooley et al. 2007; Morgan et al. 2010; Jiménez-Vicente et al. 2012), and (3) the "shape problem" in which the observed ultraviolet continuum slope is similar among AGN and softer than expected. In particular, observed SEDs typically show a break to steeper slopes around $1100 \AA$ rest wavelength (Shang et al. 2005 and references therein). For further discussion of these issues, see Lawrence (2012 and references therein).

These results have raised serious questions about the detailed applicability of standard disk models. Motivated by the availability of black hole masses in AGN estimated from broad line widths, Bonning et al. (2007, hereinafter B07) examined the optical and ultraviolet colors of QSOs in the Sloan Digital Sky Survey $(\mathrm{SDSS})^{5}$ in the context of disk theory. We defined a characteristic temperature (here denoted $T_{\text {ref }}$ ) expected on the basis of $M_{\mathrm{BH}}$ and the accretion rate $\dot{M}$ (see the detailed discussion below). Comparing $T_{\text {ref }}$ with quasar continuum colors, B07 found poor agreement between observed color trends and those predicted by standard disk models (a shift toward bluer colors

\footnotetext{
5 The SDSS Web site is http://www.sdss.org.
}

at higher temperatures). For luminosities approaching the Eddington limit, the observed colors actually become redder with increasing $T_{\text {ref }}$, in qualitative disagreement with expectations. B07 suggested that the discrepancy might result from a modification of the inner disk at radii where radiation pressure strongly affects the disk vertical structure. Modified disk models with no emission inside a critical radius gave improved agreement with observation.

In an effort to gain additional perspective on the correspondence between disk theory and observation, we here extend the results of B07 to include the QSO emission lines as diagnostics of the higher frequency portion of the disk continuum. The emission lines are governed by the intensity of the continuum at ionizing frequencies. The emitted flux at these frequencies is more sensitive to the accretion disk temperature and potentially offer a stronger test of the trend of the SED of QSOs with $T_{\text {ref }}$. We again use the large dataset of quasar spectra available from the SDSS. We compare the observed quasar spectra to model predictions computed with the code AGNSPEC (Hubeny et al. 2001) coupled with the photoionization code Cloudy (Ferland et al. 1998). With a large range of $T_{\text {ref }}$, one might expect strong trends in emission-line ratios and equivalent widths as a function of disk temperature. We find that these expectations are not borne out in observed QSO spectra.

For the sake of economy, we refer the reader to B07 for further background and references to earlier work. We assume a concordance cosmology with $\Omega_{\Lambda}=0.7, \Omega_{m}=0.3$, and $H_{0}=70 \mathrm{~km} \mathrm{~s}^{-1} \mathrm{Mpc}^{-1}$.

\section{MODELING AND DATA SAMPLING}

\subsection{Accretion Disk Properties}

In standard thin-disk theory (Shakura \& Sunyaev 1973), accreting matter gradually spirals inwards as viscous stresses transport angular momentum outward. These stresses lead to local dissipation of energy that flows vertically to the surface of the disk and is radiated by the photosphere. The resulting effective temperature, given by $F=\sigma T_{\text {eff }}{ }^{4}$, shows a radial 
dependence $T_{\text {eff }} \propto r^{-3 / 4}$ at radii substantially larger than the inner boundary of the disk. As the inner boundary is approached, the effective temperature reaches a maximum $T_{\max }$ and then drops to zero at the inner boundary if the inner boundary condition is one of zero torque. For a disk radiating locally as a blackbody, the disk spectrum is fixed by $T_{\max }$, for a given value of black hole spin. For realistic opacities, there are significant spectral features such as Lyman edges of hydrogen and helium, either in emission or absorption (Hubeny et al. 2001) that can cause disks with the same value of $T_{\max }$ to differ in their detailed continuum spectra. However, $T_{\max }$ still remains a useful sequencing parameter.

The key parameters for a QSO accretion disk are the black hole mass and spin, the accretion rate, and the observer viewing angle. An important reference luminosity is the Eddington limit,

$$
L_{\mathrm{Ed}}=\frac{4 \pi c G M_{\mathrm{BH}}}{\kappa_{e}}=\left(10^{46.10} \mathrm{erg} \mathrm{s}^{-1}\right) M_{8},
$$

where $\kappa_{e}$ is the electron scattering opacity per unit mass and $M_{8}=M_{\mathrm{BH}} /\left(10^{8} M_{\odot}\right)$. The total luminosity produced is

$$
L_{\mathrm{bol}}=\epsilon \dot{M} c^{2}=\left(10^{45.76} \mathrm{erg} \mathrm{s}^{-1}\right) \epsilon_{-1} \dot{M}_{0} .
$$

Here $\dot{M}_{0}$ is the accretion rate in $M_{\odot} \mathrm{yr}^{-1}$, and $\epsilon=10 \epsilon_{-1}$ increases from 0.057 for a non-rotating hole to 0.31 for a rapidly rotating Kerr hole with angular momentum parameter $a_{*}=0.998$ (Novikov \& Thorne 1973; Thorne 1974). For $a_{*}=$ 0.998

$$
T_{\max }=\left(10^{5.56} \mathrm{~K}\right) M_{8}^{-1 / 4}\left(L_{\mathrm{bol}} / L_{\mathrm{Ed}}\right)^{1 / 4},
$$

or alternatively, $T_{\max }=\left(10^{5.54} \mathrm{~K}\right) M_{8}^{-1 / 2} L_{46}^{1 / 4}$, where $L_{46} \equiv$ $L_{\text {bol }} /\left(10^{46} \mathrm{erg} \mathrm{s}^{-1}\right)$. For a Schwarzschild hole, $T_{\max }$ is cooler by a factor of $10^{0.46}$ for a given $M_{\mathrm{BH}}$ and $L_{\text {bol }}$ (e.g., Shields 1989). We also define a reference accretion rate $\dot{M}_{\mathrm{Ed}} \equiv$ $L_{\mathrm{Ed}} / c^{2}=\left(10^{-0.66} M_{\odot} \mathrm{yr}^{-1}\right) M_{8}$ and a dimensionless accretion rate $\dot{m} \equiv \dot{M} / \dot{M}_{\mathrm{Ed}}$. Note our definition of $\dot{M}_{\mathrm{Ed}}$ in terms of $\epsilon=1$, so that a value $\dot{m}=3.1$ gives $L_{\mathrm{Ed}}$ for $a_{*}=0.998$.

\subsection{Deriving $M_{B H}$ and $T_{r e f}$}

Following B07, we use a sequencing parameter $T_{\text {ref }}$ equal to the value of $T_{\max }$ given by Equation (3) for a given $M_{\mathrm{BH}}$ and $\dot{M}$ (assuming $a_{*}=0.998$ ). We emphasize that the difficulties described above already suggest that actual disk temperatures depart from theoretical expectations, and that $T_{\text {ref }}$ serves here as a sequencing parameter by which to look for qualitative trends that may or may not conform to the predictions of standard disk theory. Derivation of $M_{\mathrm{BH}}$ from the width of the $\mathrm{H} \beta$ or $\mathrm{Mg}$ II broad emission lines has become an accepted approximation in recent years. The FWHM of the broad lines is taken to give the circular velocity of the broad-line emitting material (with some geometrical correction factor). The radius of the broad-line region (BLR), determined from echo mapping studies, increases as a function of the continuum luminosity, $R \propto L^{\Gamma}$. Bentz et al. (2009) find $\Gamma=0.52 \pm 0.07$, consistent with the value 0.5 suggested by photoionization physics. Here we use (Shields et al. 2003)

$$
M_{\mathrm{BH}}=\left(10^{7.69} M_{\odot}\right) v_{3000}^{2} L_{44}^{0.5},
$$

where $v_{3000} \equiv \mathrm{FWHM} / 3000 \mathrm{~km} \mathrm{~s}^{-1}$, and $L_{44} \equiv$ $\lambda L_{\lambda}(5100) /\left(10^{44} \mathrm{erg} \mathrm{s}^{-1}\right)$.

The derived value of $T_{\text {ref }}$ depends almost entirely on the broad line FWHM for a given object. The bolometric luminosity can be estimated as $L_{\text {bol }}=f_{L} \times \lambda L_{\lambda}(5100)$, following Kaspi
Table 1

Properties

\begin{tabular}{ccccccc}
\hline \hline$T_{\text {ref }}$ & $M_{\mathrm{BH}}$ & $v L_{v}(5100)$ & $\dot{M}$ & $L / L_{\mathrm{Ed}}$ & $\dot{M}_{5100}$ & $\left(L / L_{\mathrm{Ed}}\right)_{5100}$ \\
\hline 5.0 & 8.72 & 44.39 & -0.95 & -1.47 & -0.77 & -1.34 \\
5.1 & 8.51 & 44.36 & -0.98 & -1.30 & -0.63 & -0.99 \\
5.2 & 8.31 & 44.33 & -0.97 & -1.13 & -0.49 & -0.65 \\
5.3 & 8.12 & 44.34 & -0.95 & -0.93 & -0.29 & -0.26 \\
5.4 & 7.90 & 44.30 & -1.00 & -0.75 & -0.12 & 0.13 \\
5.5 & 7.66 & 44.22 & -1.06 & -0.59 & -0.03 & 0.46 \\
5.6 & 7.43 & 44.13 & -1.13 & -0.44 & 0.06 & 0.78 \\
5.7 & 7.20 & 44.04 & -1.19 & -0.30 & 0.15 & 1.10 \\
\hline
\end{tabular}

Notes. Mean properties of the SDSS quasars in the various $T_{\text {ref }}$ bins. All quantities are $\log _{10}$, with $M_{\mathrm{BH}}$ in solar masses and $\dot{M}$ in solar masses per year. The subscript "5100" refers to AGNSPEC models with accretion rate adjusted to match observed continuum at $5100 \AA$. See the text for discussion.

et al. (2000). Using Equation (4) and this expression for $L_{\mathrm{bol}}$, Equation (3) becomes

$$
T_{\text {ref }}=\left(10^{5.43} \mathrm{~K}\right) v_{3000}^{-1} L_{44}^{-(\Gamma-0.5) / 2}\left(f_{L} / 9\right)^{-1 / 4} .
$$

We use a bolometric correction factor $f_{L}=9$ (Kaspi et al. 2000; Richards et al. 2006).

\subsection{SDSS Spectra and $T_{\text {ref }}$ Bins}

We use the SDSS DR5 spectroscopic data base for "quasars" as our primary observational material. We selected AGN using an automated spectrum fitting program and imposing quality cuts as described by Salviander et al. (2007). These fits give values for quantities such as the flux and width of the broad $\mathrm{H} \beta$ line and the continuum flux at various rest wavelengths including $\lambda 5100$. The adopted redshift range is $z=0.1-0.8$ so as to keep the [O III] nebular lines out of the telluric water vapor region. Approximately one-half of the objects fall above the QSO/Seyfert galaxy luminosity boundary at $\log v L_{v}(5100)=$ 44.44 in units of $\mathrm{erg} \mathrm{s}^{-1}$. Following B07, we derive $M_{\mathrm{BH}}$ and $T_{\text {ref }}$ for the individual quasars as described above, and then group them into bins with $\log T_{\text {ref }}$ ranging from 5.0 to 5.7 defined by windows of 4.95 to 5.05 , etc. Table 1 gives the average values of $\log M_{\mathrm{BH}}$ and $\log v L_{v}(5100)$ for these bins. Also given is the accretion rate and resulting $\log T_{\text {ref }}$ on the assumption $f_{L}=9$ and $a_{*}=0.998$, based on the bin averages for $\log M_{\mathrm{BH}}$ and $\log v L_{v}(5100)$. (An alternative approach to the accretion rate is discussed below.) The values of $\log M_{\mathrm{BH}}$ systematically decrease from 8.27 to 7.20 as $\log T_{\text {ref }}$ increases from 5.0 to 5.7. The mean luminosity and accretion rate differs only slightly among the bins. As a result, the Eddington ratio increases with $\log T_{\text {ref }}$ from $\log L / L_{\mathrm{Ed}}=-1.5$ to -0.3 across the bins.

For each of the bins, we form a composite spectrum giving equal weight to all objects based on the frequency-integrated flux at earth over the spectrum. These composite spectra were measured to give values of several observational quantities (e.g., the $[\mathrm{Ne} \mathrm{v}$ ] emission-line intensity) used in the analysis below. Line measurements were carried out in IRAF. ${ }^{6}$

Note that the high values of $T_{\text {ref }}$ given by Equation (5) for the SDSS quasars already conflict with observed SEDs. As noted above, SEDs typically turn over around 1100 A. Observed EUV energy distributions fall far below the predictions of blackbody

\footnotetext{
6 IRAF is distributed by the National Optical Astronomy Observatories, which are operated by the Association of Universities for Research in Astronomy, Inc., under cooperative agreement with the National Science Foundation.
} 


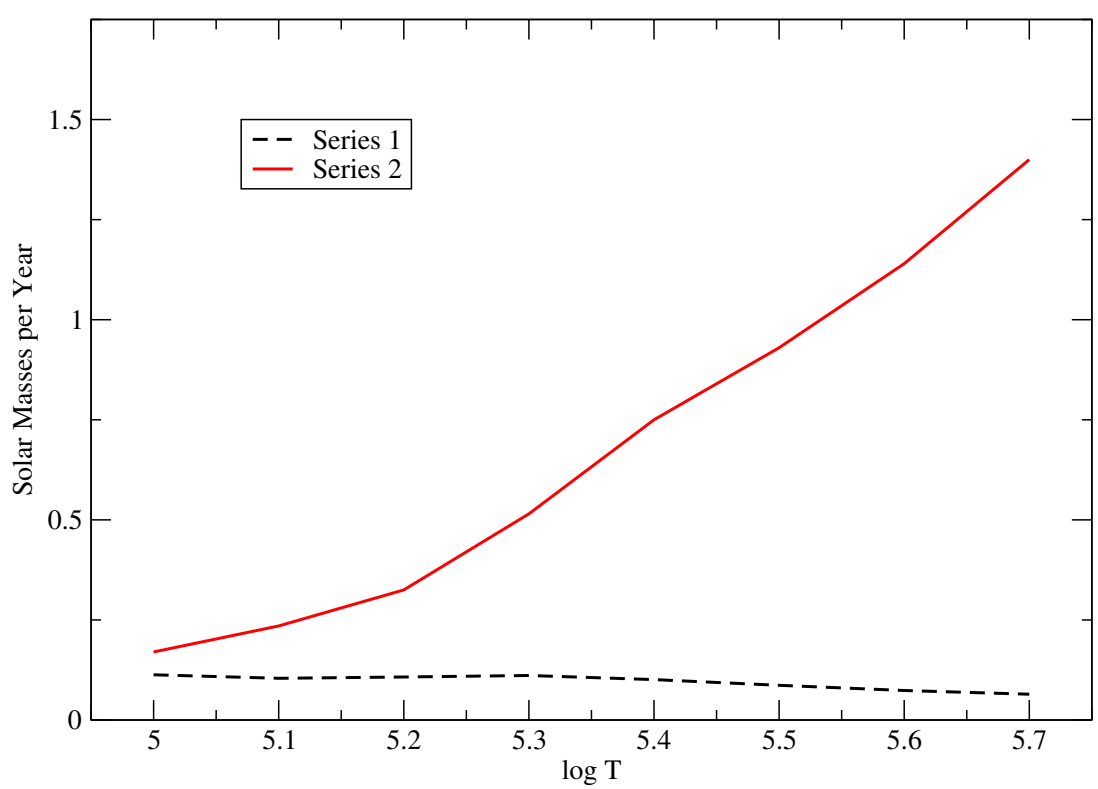

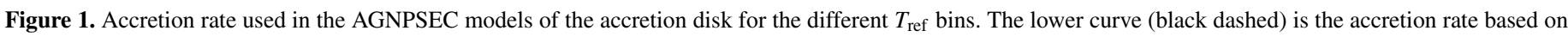

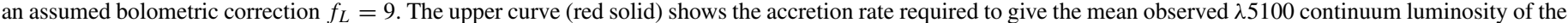

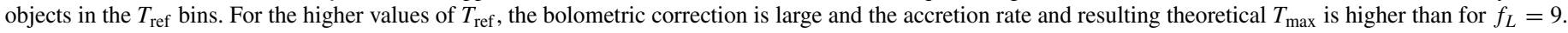
This aggravates the discrepancy between predicted and observed emission-line properties, as shown in subsequent figures.

(A color version of this figure is available in the online journal.)

or disk models for such high temperatures, as illustrated in Figure 1 of Lawrence (2012).

\subsection{Accretion Disk Models}

Our goal is to compare basic trends in observed and predicted AGN properties as a function of $T_{\text {reff }}$. To this end, we have computed disk models using the AGNSPEC program (Hubeny et al. 2001) in the manner described in B07. These models take as input parameters the black hole mass $M_{\mathrm{BH}}$ and spin parameter $a_{*}$, accretion rate $\dot{M}$, viscosity parameter $\alpha$, and inclination $i$ of the disk axis to the line of sight. For simplicity, we adopt the commonly assumed value $\alpha=0.1$ and focus on rapidly rotating black holes with $a_{*}=0.998$ (Thorne 1974). We take $\cos i=0.6$ as representative of randomly oriented AGN subject to an exclusion of inclinations near edge-on because of the dusty torus postulated in the unified model of AGN (Antonucci $\&$ Miller 1985). The AGNSPEC code computes the vertical gravity and locally emitted flux $F(R)$ in the orbiting frame, and calculates for each radius the vertical structure and emergent spectrum with the aid of a pre-computed grid of models. The spectrum observed at infinity is summed over all emitting radii accounting for all relativistic effects. In order to avoid an excessive number of free parameters, we restrict our models to the case $a_{*}=0.998$. The spin of the black holes in AGN has been much debated, with some authors arguing for moderate values based on likely merger histories or fitting of disk models to the SEDs of individual AGN (e.g., Czerny et al. 2011 and references therein).

\section{PROBLEMS WITH THE STANDARD DISK MODEL}

Here we discuss tests of the success of the standard thin accretion disk model to explain a variety of observed properties of AGN. We discuss two different approaches for determining the accretion rate of the disk, which, together with $M_{\mathrm{BH}}$, determines $T_{\text {ref }}$. First, we follow B07 in assuming a universal bolometric correction $f_{L}=9$ as described above. Then we discuss an alternative approach in which $\dot{M}$ is adjusted in the disk models so as to reproduce the observed value of $v L_{v}(5100)$ (cf. Figure 1).

\subsection{Emission-line Intensities}

As found above, typical values of $T_{\max }$ for the accretion disk (for the parameters of our sample) are of the order of 200,000 K, corresponding to a blackbody peak at $h v \approx 3 k T \approx 1.5 h \nu_{\mathrm{H}}$, where $h v_{\mathrm{H}}=I_{\mathrm{H}}$ is the hydrogen ionization potential. Thus, the output of the disk in hydrogen ionizing photons, $Q\left(\mathrm{H}^{0}\right)$, will be sensitive to the value of $T_{\text {ref }}$, and the sensitivity will be greater for ions with higher ionization potentials. The amount of ionized gas and the resulting line luminosities, as well as the relative intensities of lines from different levels of ionization, should depend strongly on $T_{\text {ref. }}$. If the actual disk temperature is less than $T_{\text {ref }}$, the sensitivity of the ionizing output to any differential changes in that temperature as a function of $T_{\text {ref }}$ will be even greater.

\subsubsection{H $\beta$ Equivalent Width}

A basic application of this concept is the equivalent width of the broad $\mathrm{H} \beta$ line. For a photoionized nebula, the luminosity of the $\mathrm{H} \beta$ line is given by radiative recombination theory (Osterbrock \& Ferland 2006) as

$$
L(\mathrm{H} \beta)=(\Omega / 4 \pi)\left(\epsilon_{\mathrm{H} \beta} / \alpha_{\mathrm{B}}\right) \mathrm{Q}\left(\mathrm{H}^{0}\right) .
$$

Here, $\Omega / 4 \pi$ is the covering fraction for the BLR, $\epsilon_{\mathrm{H} \beta}$ is the recombination-line emission coefficient, $\alpha_{B}$ is the radiative recombination coefficient for hydrogen to quantum levels $n=2$ and higher, and $Q\left(\mathrm{H}^{0}\right)$ is the ionizing photon luminosity (photons per second). The $\mathrm{H} \beta$ equivalent width is then given by $\mathrm{EW}=L(\mathrm{H} \beta) / \mathrm{L}_{\lambda}(4861 \AA)$. As $T_{\max }$ increases, we expect a strong increase in $\mathrm{EW}(\mathrm{H} \beta)$, because the ionizing frequencies are more sensitive to disk temperature than the optical continuum emission. Figure 2 shows the predicted EW for the set of 




Figure 2. Predicted and observed equivalent width of broad $\mathrm{H} \beta$ line as a function of $T_{\max }$ (models) or $T_{\text {ref }}$ (observed). The SDSS composites show a systematic decrease in EW with increasing $T_{\text {ref }}$, in contrast to the strong increase predicted by the standard disk model for the corresponding values of $T_{\max }$. The modified disk model shows a leveling off of the EW for higher $T_{\max }$ although at an excessively large value; the discrepancy in slope remains at lower $T_{\max }$.

(A color version of this figure is available in the online journal.)

AGNSPEC models described above, on the assumption of a covering fraction of 0.5 independent of $T_{\max }$. The expected increase of $\mathrm{EW}$ with $T_{\max }$ is evident. (The covering fraction of 0.5 was chosen to give agreement with the observed $\mathrm{EW}$ of $\mathrm{H} \beta$ at $\log T_{\text {ref }}=5.0$ and is reasonable in the context of the unified model of AGN.)

Figure 2 also shows the observed trend of the $\mathrm{H} \beta \mathrm{EW}$ as a function of $T_{\text {ref }}$ for our SDSS composites. The observed values show a progressive decrease from $120 \AA$ to $50 \AA$ as $\log T_{\text {ref }}$ increases from 5.0 to 5.7. This trend is in qualitative disagreement with the model prediction. While it is possible that the covering fraction changes systematically with $T_{\text {ref }}$, an order-of-magnitude decrease in $\Omega / 4 \pi$ would be required from $\log T_{\text {ref }}=5.0$ to 5.7. A search for independent signatures of such a trend may be worthwhile. However, it seems likely that such an extreme trend in covering fraction would have been noticed in prior investigations. Therefore, the discrepancy shown in Figure 2 reinforces the case that something is wrong with the standard disk model in regard to the ionizing photon output. This discrepancy resembles that found in B07 in which the continuum colors do not show the expected trend toward bluer colors at higher $T_{\text {ref }}$. The systematic decrease in $\mathrm{H} \beta$ equivalent width with increasing $T_{\text {ref }}$ gives a new context to the long known correlation between the EW and FWHM of the broad $\mathrm{H} \beta$ emission line (Boroson \& Green 1992), since the derived $T_{\text {ref }}$ depends mainly on the $\mathrm{H} \beta$ broad line width (see Equation (5)). While there were already reasons to doubt that AGN disks achieve temperatures as high as $T_{\text {ref }}$, it is striking that the observed equivalent width actually trends in a direction contrary to the expected trend with $T_{\text {ref }}$.

Equation (6) does not allow for collisional excitation or other processes that can affect $\mathrm{H} \beta$ in the BLR. In order to estimate the magnitude of these effects, we computed a series of models for the BLR using version 10.0 of the Cloudy photoionization code, most recently described by Ferland et al. (1998). The input ionizing spectrum was the output of the AGNSPEC program as described above. Following Ferland et al. (2009), the BLR was modeled as an optically thick slab with hydrogen density
$N_{\mathrm{H}}=10^{10} \mathrm{~cm}^{-3}$ and incident ionizing flux $\phi=10^{19} \mathrm{~cm}^{-2} \mathrm{~s}^{-1}$, giving an ionization parameter $U=\phi / N c=10^{-1.5}$. Equation (6) corresponds to a predicted flux $F(\mathrm{H} \beta)=\left(\epsilon_{\mathrm{H} \beta} / \alpha_{\mathrm{B}}\right) \phi$. The model results exceeded this prediction by 0.08 and $0.17 \mathrm{dex}$ for $\log T_{\max }=5.0$, respectively. These values and their trend with $T_{\max }$ do not alter the basic conclusions reached above.

\subsubsection{Ionization Ratios}

Typical AGN spectra show emission lines from ions with a large range of ionization potentials. In the case of ionized nebulae, a useful diagnostic of the ionizing continuum is the ratio of the lines of $\mathrm{He}$ II and $\mathrm{H}$ I. In a normal nebular ionization structure, He II occupies an inner volume of the Strömgren sphere (or a surface layer of a slab) whose fraction of the total $\mathrm{H}^{+}$volume is proportional to the ionizing photon luminosity ratio, $Q\left(\mathrm{He}^{+}\right) / Q\left(\mathrm{H}^{0}\right)$. Here $Q\left(\mathrm{He}^{+}\right)$is the ionizing photon luminosity above 4 Rydbergs frequency. The recombination line ratio $I(\mathrm{He}$ II $\lambda 4686) / I(\mathrm{H} \beta)$ is in turn proportional to the nebular average of $N\left(\mathrm{He}^{+2}\right) / N\left(\mathrm{H}^{+}\right)$, giving

$$
I(\lambda 4686) / I(\mathrm{H} \beta)=1.02 \mathrm{Q}\left(\mathrm{He}^{+}\right) / \mathrm{Q}\left(\mathrm{H}^{0}\right) .
$$

This assumes that the nebula is optically thick to the Lyman continuum.

Figure 3 shows that $Q\left(\mathrm{He}^{+}\right) / Q\left(\mathrm{H}^{+}\right)$does indeed increase strongly with increasing $T_{\max }$ in the AGNSPEC models. Figure 4 shows the composite spectra for the various bins in $\log T_{\text {ref. The }}$ broad He II line is blended with Fe II and difficult to measure. The He II line in the narrow line spectrum is well defined for our cooler composites, in which the broad line widths are large. However, it is difficult to separate the narrow from the broad components of $\lambda 4686$ for the hotter composites, which have relatively narrow broad lines. Nevertheless, two points can be made. A subjective inspection of the figure indicates little trend in the narrow He II strength as a function of $\log T_{\text {ref. }}$ Also, the observed values of narrow $\mathrm{He}$ II $/ \mathrm{H} \beta$ for the lower $T_{\text {ref }}$ bins are 


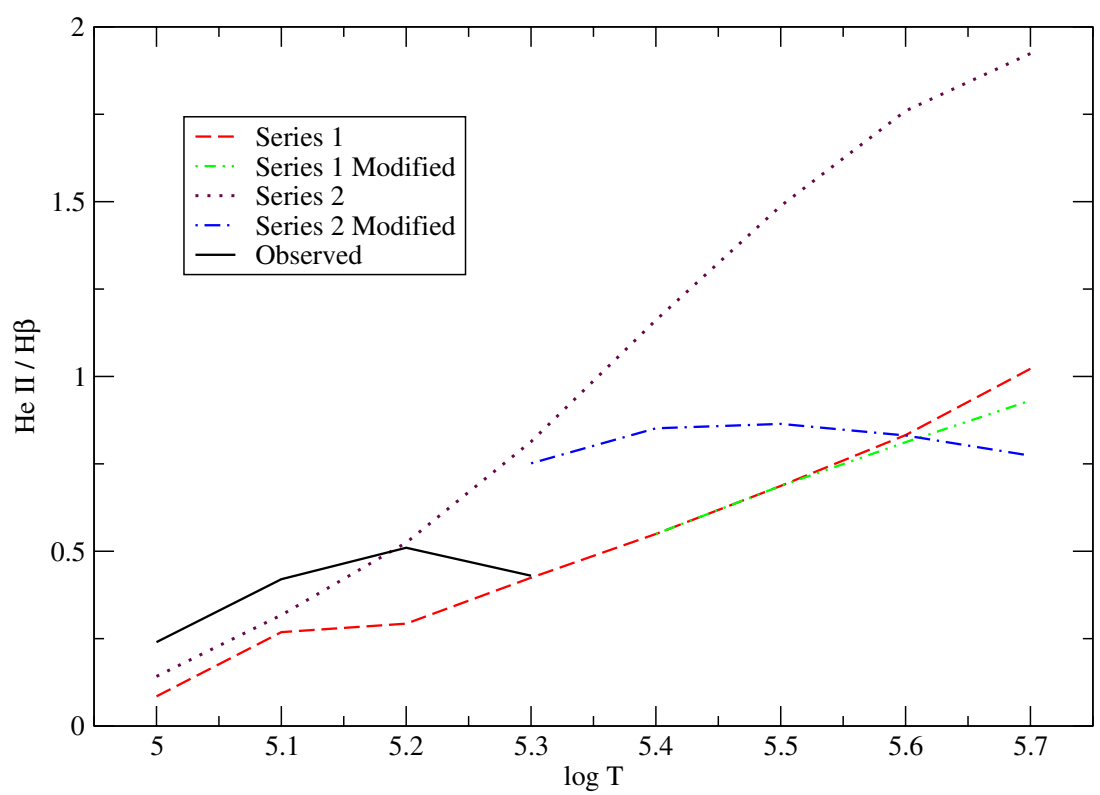

Figure 3. Predicted line intensity ratio of $\mathrm{He} \mathrm{II} / \mathrm{H} \beta$ for accretion disk model compared with observed ratio for the narrow lines. The horizontal axis is $T_{\max }$ for the models and $T_{\text {ref }}$ for the observed values. Note the strong underprediction of He II for the lowest temperatures and the strong trend in the model predictions in contrast to the observed intensities. The He II narrow line intensity is difficult to separate from the broad line profile for higher $T_{\max }$. See the text for discussion.

(A color version of this figure is available in the online journal.)
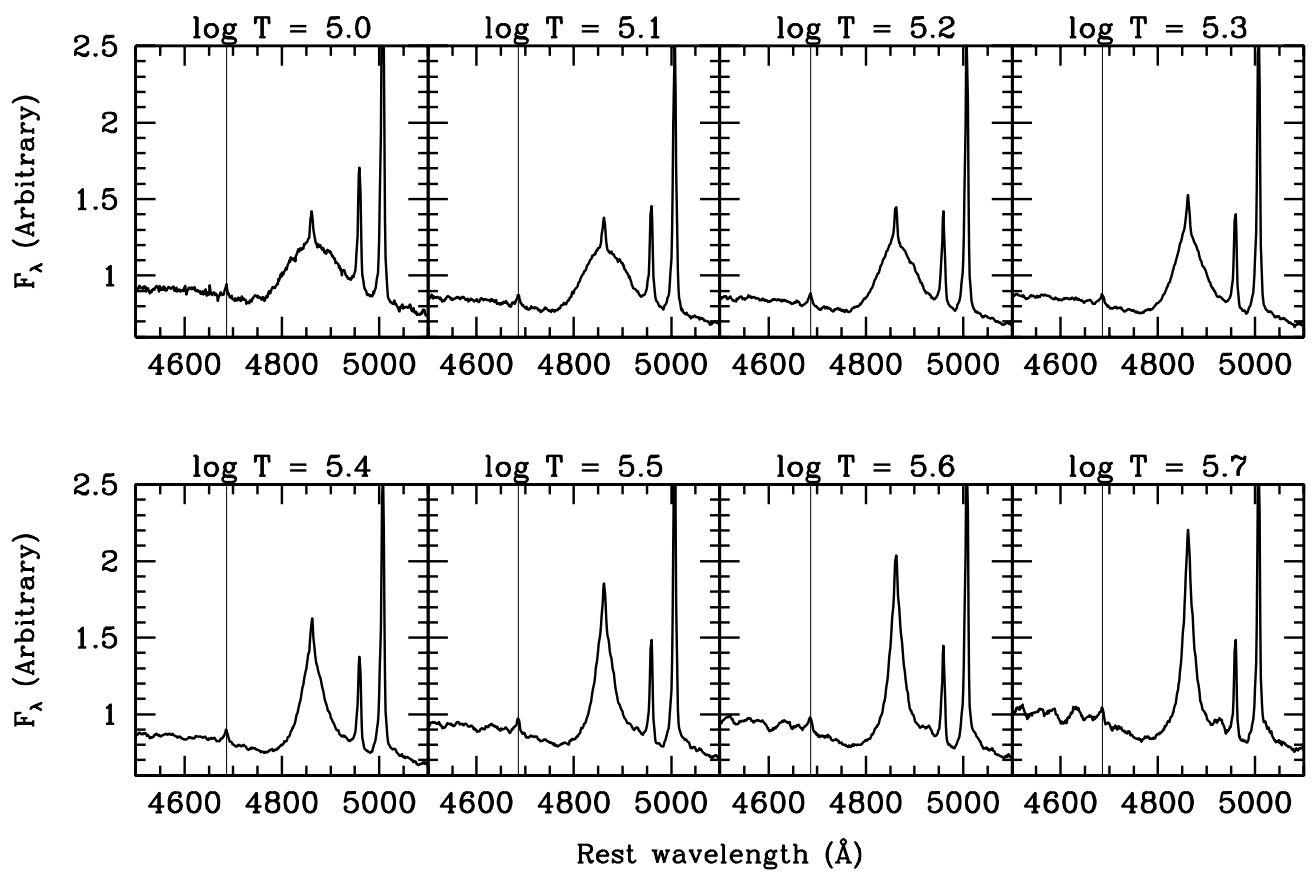

Figure 4. Composite spectra of SDSS quasars binned by $T_{\text {ref }}$. As described in the text, $T_{\text {ref }}$ is the accretion disk temperature predicted on the basis of standard disk theory, not a measurement of the actual disk temperature. The thin vertical line represents the wavelength of the He II $\lambda 4686$ line.

larger than would be expected for the predicted disk continuum, absent an additional hard component such as a power-law; and there is little trend with increasing $T_{\text {ref }}$.

For an observationally more feasible test, we consider the narrow line ratio of $[\mathrm{Ne} \mathrm{v}] \lambda 3426$ to $[\mathrm{Ne} \mathrm{III}] \lambda 3869$. The $\mathrm{Ne}^{+4}$ ion requires even higher ionizing photon energies than $\mathrm{He}^{+2}$ and thus the $[\mathrm{Ne} \mathrm{v}] /\left[\mathrm{Ne}\right.$ III] ratio should be even more sensitive to $T_{\max }$ than $\mathrm{He}$ II $/ \mathrm{H} \beta$. For illustration, we computed a series of Cloudy models for the Narrow Line Region (NLR). The input ionizing spectrum was the AGNSPEC continuum for $T_{\max }$ corresponding to the various adopted values of $T_{\text {ref }}$. The NLR was modeled as an optically thick slab with hydrogen density $N_{\mathrm{H}}=10^{4.0} \mathrm{~cm}^{-3}$ and incident ionizing flux $\phi=10^{12.5} \mathrm{~cm}^{-2} \mathrm{~s}^{-1}$, giving an ionization parameter $U=\phi / N c=10^{-2.0}$. The default chemical abundances were used, including $\log N(\mathrm{~A}) / N(\mathrm{H})=$ $-1.00,-3.31,-4.0$ for $\mathrm{He}, \mathrm{O}$, and $\mathrm{Ne}$, respectively. Figure 5 shows the predicted $[\mathrm{Ne} \mathrm{v}] /\left[\mathrm{Ne}\right.$ III] ratio as a function of $T_{\max }$. The models show the expected strong trend with $T_{\max }$, with $[\mathrm{Ne} \mathrm{v}]$ being very weak for the cooler models.

However, the observed values of $[\mathrm{Ne} \mathrm{V}] /[\mathrm{Ne} \mathrm{III}]$ show no significant trend with $T_{\text {ref. }}$. Thus, this observed line ratio, involving emission lines from the NLR rather than the BLR, 


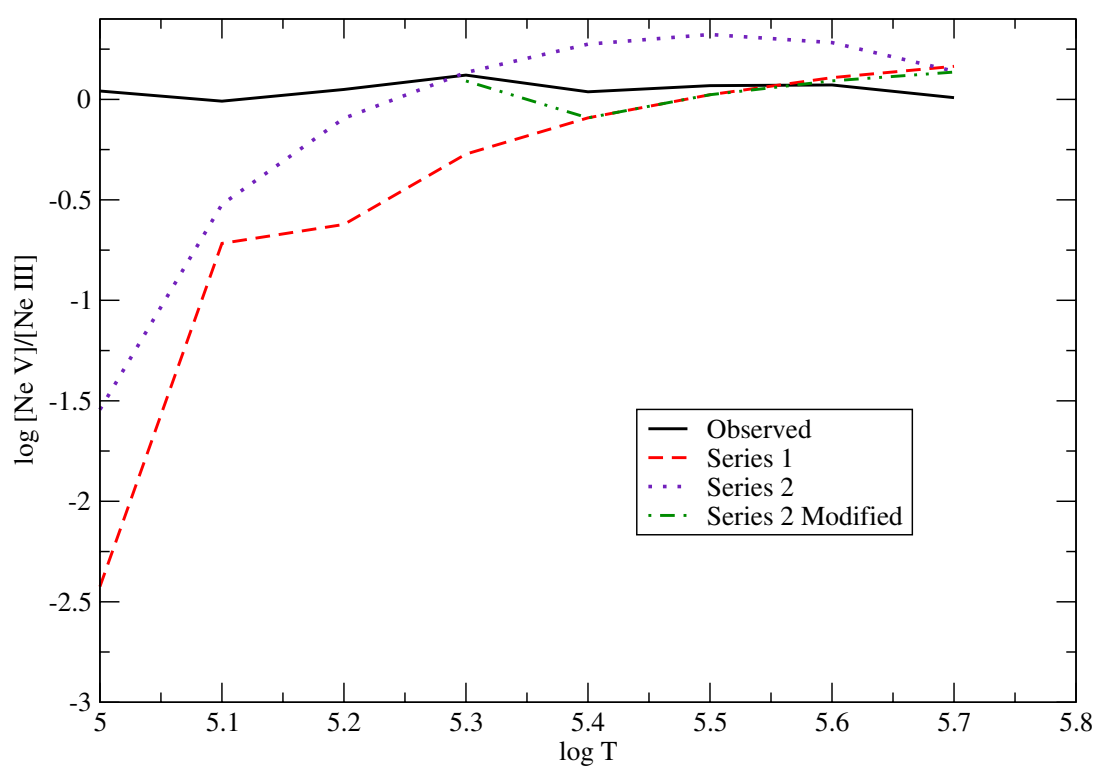

Figure 5. Predicted line intensity ratio of $\mathrm{Ne} \mathrm{v/Ne} \mathrm{III} \mathrm{for} \mathrm{accretion} \mathrm{disk} \mathrm{model} \mathrm{compared} \mathrm{with} \mathrm{observed} \mathrm{ratio} \mathrm{as} \mathrm{a} \mathrm{function} \mathrm{of} T_{\max }$ (models) or $T_{\text {ref }}$ (observed). Note the strong trend in the model prediction that is not reflected in the observed intensities. See text for discussion.

(A color version of this figure is available in the online journal.)



Figure 6. Predicted optical to soft X-ray spectral index $\alpha_{\mathrm{os}}$ from AGNSPEC models compared with observed values derived from Laor et al. (1997). The horizontal axis is $T_{\max }$ for the models and $T_{\text {ref }}$ for the observed values. Note the absence of trend with $T_{\text {ref }}$ for the observed values. See the text for discussion.

(A color version of this figure is available in the online journal.)

also fails to support the expectation of a harder spectrum in the ionizing ultraviolet for higher $T_{\text {ref }}$.

\subsection{Soft X-Rays}

AGN spectra characteristically include emission in the hard and soft X-ray bands. In general, this emission involves frequencies too high to be the result of ordinary thermal continuum emission from a standard accretion disk. Laor et al. (1997) and others have noted the inconsistency of observed soft X-ray fluxes with the simple thin-disk model. A Comptonizing corona, producing a power-law spectrum extending to high photon energies, is sometimes invoked (Czerny \& Elvis 1987). However, for the higher values of $T_{\text {ref }}$ that we consider here, substantial soft $\mathrm{X}$-ray emission from the inner disk is predicted, and there is the possibility of overproducing soft X-rays. We have computed $T_{\text {ref }}$ in the manner described above, for the AGN in the sample described by Laor et al. (1997). Laor gives X-ray fluxes and values of the optical (3000 $\AA$ ) to soft X-ray $(0.3 \mathrm{keV})$ spectral index defined by $L_{\mathrm{s}} / L_{\mathrm{o}}=\left(v_{s} / v_{o}\right)^{\alpha_{\mathrm{os}}}$. Figure 6 shows the predicted value of $\alpha_{\mathrm{os}}$ as a function of $T_{\max }$ for a simple set of AGNSPEC models with $M_{\mathrm{BH}}=10^{8} M_{\odot}$. The increase in $\alpha_{\mathrm{os}}$ with increasing $T_{\text {ref }}$ is evident. In contrast, the observed values of $\alpha_{\text {os }}$ in the Laor sample, grouped into our same bins in $T_{\text {ref }}$, show no significant trend with $T_{\text {ref }}$. In particular, for $\log T_{\text {ref }}>5.2$, the models predict a greater value of $L_{\mathrm{s}} / L_{\mathrm{o}}$ than observed. This is significant, because one might invoke a Comptonized component to explain the soft X-rays for low $T_{\max }$; but the hotter disks, in the standard model, overpredict the soft X-rays even before 
any Comptonized component is added. While the origin of the $\mathrm{X}$-ray flux of AGN remains uncertain, it is remarkable that the observed values of $\alpha_{\text {os }}$ show no trend with $T_{\text {ref }}$. Thus, we have in $\alpha_{\text {os }}$ yet another qualitative discrepancy between observed AGN properties and the predictions of the simple disk model.

The dependence of $\alpha_{\mathrm{os}}$ on disk effective temperature has been previously discussed by other authors. For example, Deroches et al. (2009) in their Figure 6 show $\alpha_{\mathrm{os}}$ versus $\log \left[\left(L_{\mathrm{bol}} / L_{\mathrm{Ed}}\right)^{1 / 4} M_{\mathrm{BH}}{ }^{1 / 4}\right]$. Aside from minor differences in the derivation of $L_{\text {bol }}$ and $M_{\mathrm{BH}}$, their temperature axis can be calibrated to our $\log T_{\text {ref }}$ scale by adding 7.56. The SDSS objects in their plot span a similar range in $T_{\max }$ to ours, and likewise show little systematic trend in $\alpha_{\mathrm{os}}$ with $T_{\text {ref }}$.

\subsection{Models with Variable Bolometric Correction}

As noted above and in B07, the use of a universal bolometric correction $f_{L}=9$ is not consistent with the proposition that disk temperatures may differ considerably among AGN. An alternative approach is to tune the value of $\dot{M}$ in the AGNSPEC models so as to give the observed value of $\lambda L_{\lambda}(5100)$ for a given $T_{\text {ref }}$ bin. We have computed a set of models in this fashion (shown in Figure 1), still assuming $a_{*}=0.998$ and $\mu=0.6$. We find that for $\log T_{\max }=5.0$, the resulting model has a value $L_{\mathrm{bol}}$ giving $f_{L} \approx 10$, so that the model differs little from the $f_{L}=9$ case. However, the value of $f_{L}$ implied by the AGNSPEC fits increases progressively to a value of $f_{L} \approx 50$ for $\log T_{\max }=5.7$. These models involve very large accretion rates which exceed the more conventional $\dot{M}$ based on $f_{L}=9$ by nearly an order of magnitude. Such large accretion rates put increased strain on available accretion supplies. They also give $L_{\text {bol }}$ much larger than $L_{\mathrm{Ed}}$, so that the inner radii of such disks would likely involve serious modifications due to radiation pressure and mass loss, as discussed below. Nevertheless, we start our discussion of these models by revisiting the several observational tests above using the predicted disk spectra from AGNSPEC.

We use $T_{\text {ref }}$ to refer to the original SDSS quasar bins, even though the actual $T_{\max }$ of the models is now higher. These models are useful, nevertheless, because we may think of the nominal $\log T_{\text {ref }}$ spectrum bins as a sequencing procedure that gives us a set of SDSS AGN involving a large range in predicted $T_{\max }$, even if there are ambiguities as to how well the nominal value agrees with the actual disk temperature. Because the models adjusted to $L_{5100}$ have a greater range of $\dot{M}$ and $\log T_{\max }$ than the nominal models, they are expected to show even stronger trends in observational quantities sensitive to $T_{\max }$, such as continuum color and high ionization emission-line strength.

Figure 2 shows the predicted equivalent width of $\mathrm{H} \beta$ for the luminosity-fitted models. The strong increase with the bin value of $\log T_{\text {ref }}$ is now even more pronounced and in greater contrast to the observed decrease. Figures 3 and 5 show the predicted $\mathrm{He}$ II and $[\mathrm{Ne} \mathrm{V}]$ intensity. The strong increase in [ $\mathrm{Ne} \mathrm{V}]$ with $T_{\max }$ is now in even greater contrast to the observed constancy of [Ne v]/[Ne III]. Figure 6 shows the extreme trend in $\alpha_{\text {os }}$ versus $T_{\max }$ for the luminosity-fitted models. The hotter cases greatly overpredict the observed soft X-ray fluxes.

Clearly, the luminosity-fitting approach only exacerbates the discrepancy between the theoretical predictions and observations. However, this approach may have merit physically, since it avoids the use of a universal bolometric correction. Moreover, for the higher values of $T_{\text {ref }}$, the $f_{L}=9$ models severely underpredict the luminosity at $\lambda 5100$. If the accretion rate is not increased, some restructuring of the AGN continuum source is needed to channel energy more efficiently into the continuum at optical wavelengths. In any case, the standard thin-disk model requires some kind of modification to explain the continuum and emission-line observations.

\section{A MODIFIED DISK MODEL}

B07 found that observed continuum colors of SDSS QSOs did not become progressively bluer with increasing $T_{\text {ref }}$ in the manner predicted by the AGNSPEC models. They suggested that this resulted from higher Eddington ratios at higher $T_{\text {ref }}$, leading to departures from the standard thin disk model. This might involve thickening by radiation pressure, inefficient "slim disk" accretion, and mass loss in a radiation-driven wind (see also Deroches et al. 2009). As an estimate of the potential effect on the continuum colors, we computed AGNSPEC models in which the inner disk was truncated inside the radius where radiation pressure gave a vertical scale height $H$ for the disk, relative to the radius, of $H / R=0.5$. This led to improved agreement with the observed colors, in particular giving a nonmonotonic trend of color with $T_{\max }$.

Here we explore a more physical model in which the disk effective temperature is modified inside the critical radius. The basic idea, following Poutanen et al. (2007) and Begelman et al. (2006), is to take into account the luminosity produced at a given radius relative to $L_{\mathrm{Ed}}$. If the luminosity emitted by a wide annulus, $d \ln L / d \ln R$, is well below $L_{\mathrm{Ed}}$, then the disk will not be severely thickened by radiation pressure, and standard disk physics applies. However, except near the inner boundary, $d \ln L / d \ln R$ increases with decreasing $R$. For sufficiently large values of $\dot{M} / M_{\mathrm{BH}}$, a radius is reached where $d \ln L / d \ln R$ reaches $L_{\mathrm{Ed}}$. Near this radius, serious modifications of the disk structure can be anticipated. Shakura \& Sunyaev (1973) called this the "spherization radius" $R_{\mathrm{sp}}$ on the basis that radiation pressure would severely thicken the disk. Poutanen et al. (2007) find that, inside $R_{\mathrm{sp}}$, a combination of wind mass loss and advection occurs to a degree that keeps the local luminosity $d \ln L / d \ln R$ close to $L_{\mathrm{Ed}}$. This results in a break in the run of effective temperature with radius, so that inside $R_{\text {sp }}$ one has $T_{\text {eff }} \propto R^{-1 / 2}$ rather than the standard dependence $T_{\text {eff }} \propto R^{-3 / 4}$. This reduces the value of $T_{\max }$ actually reached in the inner disk as well as the luminosity radiated from the hottest parts of the disk.

The value of $R_{\mathrm{sp}}$ in units of the gravitational radius is approximately $r_{\mathrm{sp}} \equiv R_{\mathrm{sp}} / R_{\mathrm{g}} \approx \dot{m}$. As $\dot{M}$ increases, the effective temperature at $R_{\text {sp }}$ actually decreases, following

$$
T_{\mathrm{sp}}=\left(10^{5.75} \mathrm{~K}\right) \dot{m}^{-1 / 2} M_{8}^{-1 / 4}
$$

Here we have used the Newtonian expression for the local flux, omitting the normal factor of three increase in flux due to viscous transport of energy from smaller radii. This modification was found by Poutanen et al. (2007) because of energy loss in the inner disk due to advection and mass loss. For large values of $\dot{m}$, a luminosity $\sim L_{\mathrm{Ed}}$ is emitted in the vicinity of $R_{\mathrm{sp}}$, and a similar luminosity is emitted per unit $\ln R$ at smaller radii. Thus $T_{\mathrm{sp}}$ substantially controls the character of the emitted spectrum. Hence, the fact that $T_{\text {sp }}$ decreases with increasing $\dot{m}$ once spherization comes into play is suggestive of the reversal in the trend of colors with $T_{\text {ref }}$ found in B07. (Note that, in this context, $T_{\max }$ is defined in terms of the accretion rate entering the disk at large radii, and differs from the actual maximum temperature in the inner disk.)

The potential for an inverse relationship between $\dot{M}$ and disk color temperature is strengthened if one considers the optical 

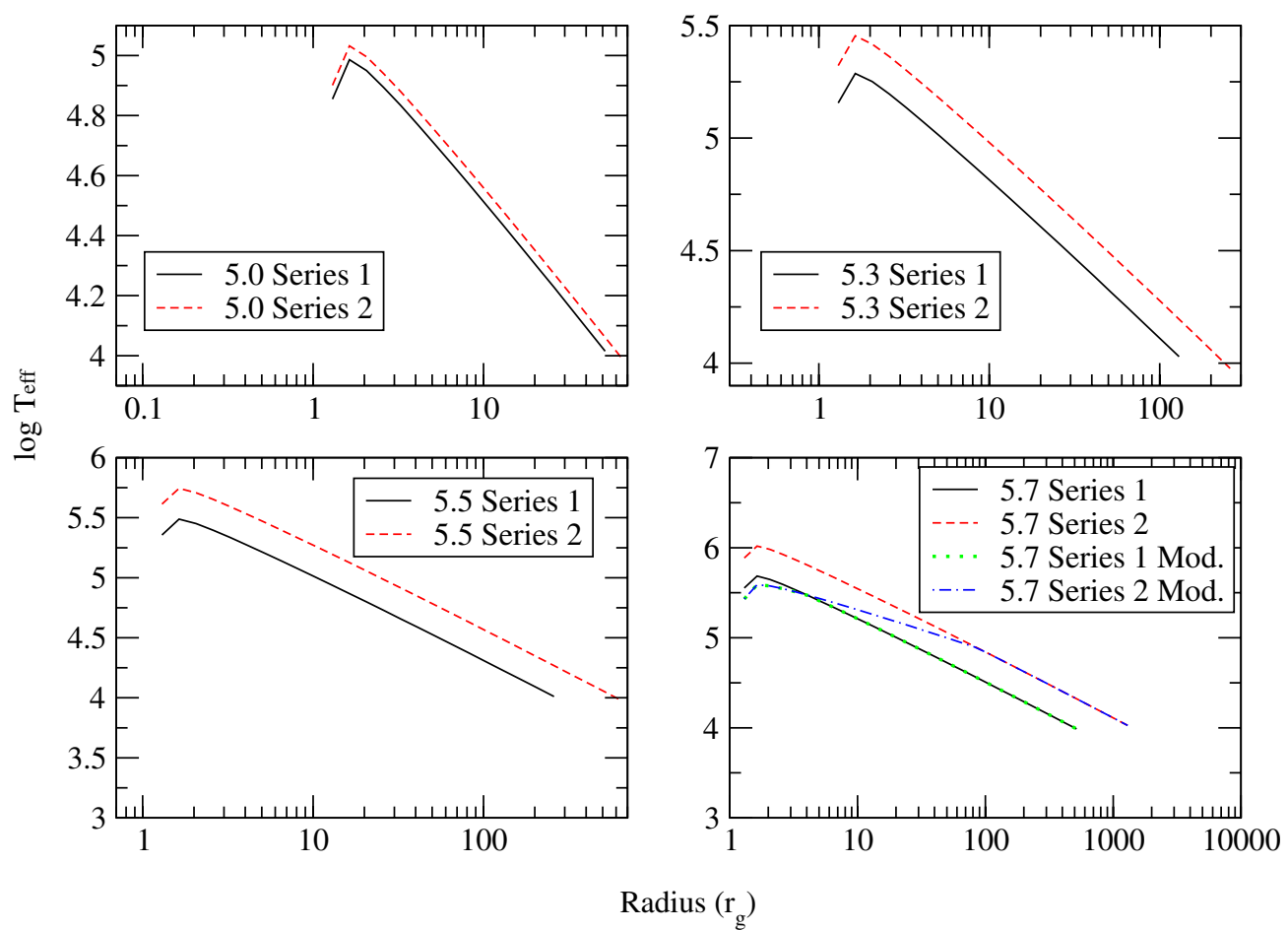

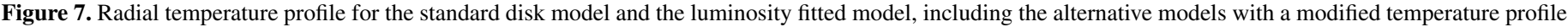
in the inner disk. See the text for discussion.

(A color version of this figure is available in the online journal.)

depth of the disk wind. In the case of highly super-Eddington accretion, Poutanen et al. (2007) find that a large part of the inflowing material is expelled as a wind in the vicinity of $R_{\mathrm{sp}}$. In the simple approximation of a spherical wind with constant radial velocity equal to the circular orbital velocity at $R_{\mathrm{sp}}$, the optical depth of the wind, measured radially from $R_{\mathrm{sp}}$, is $\tau_{\mathrm{sp}} \approx \dot{m}^{1 / 2}$. The optical depth in the wind, measured outward from some radius $R$ that exceeds $R_{\mathrm{sp}}$, is $\tau(>R) \approx \tau_{\mathrm{sp}}\left(R / R_{\mathrm{sp}}\right)^{-1}$. For the radius of the photosphere, where $\tau(>R)=1$, this gives $r_{\mathrm{ph}} \approx \dot{m}^{3 / 2}$. The effective temperature at the photosphere is

$$
T_{\mathrm{ph}}=\left(10^{5.75} \mathrm{~K}\right) \dot{m}^{-3 / 4} m_{8}^{-1 / 4} .
$$

This raises the prospect that, for super-Eddington accretion rates, the maximum visible disk temperature will be $T_{\mathrm{ph}}$ rather than $T_{\max }$. An estimate of the progression of the disk temperature as it actually appears to the observer is then

$$
T_{\text {app }}=\min \left(T_{\max }, T_{\mathrm{ph}}\right) .
$$

This assumes that there is enough absorption opacity in the wind to reprocess the emerging energy; otherwise, the escaping spectrum may still be characterized by $T_{\mathrm{sp}}$.

For our Series 1 models above, with an accretion rate based on $f_{L}=9$, the value of $\log L / L_{\mathrm{Ed}}$ reaches -0.3 for $\log T_{\max }=5.7$. Thus, unless opacity sources other than electron scattering reduce the effective Eddington limit, the effects of radiation pressure may be modest. However, for our Series 2 models with $\dot{M}$ adjusted to fit the continuum at $\lambda 5100, \log L / L_{\text {Ed }}$ reaches +1.1 for $\log T_{\max }=5.7$. For this series, at $\log T_{\max }=(5.0,5.4,5.7)$ we have $\log \dot{m}=(-0.79,+0.29,+1.65) ; \log \min \left(T_{\max }, T_{\mathrm{sp}}\right)=$ $(5.05,5.44,5.13)$, and $\log \min \left(T_{\max }, T_{\mathrm{ph}}\right)=(5.05,5.37,4.75)$. Thus, a reversal of the trend of the apparent temperature of the disk as a function of the nominal $T_{\max }$ would be expected at $\log T_{\max } \approx 5.4$. The fact that this value agrees with the turnaround in the color trend found in B07 is fortuitous, given the approximations in our discussion. However, this simple calculation does illustrate the possibility of a reverse trend of color temperature with $\dot{M} / M_{\mathrm{BH}}{ }^{2}$ for super-Eddington rates.

In order to explore the impact of super-Eddington accretion on the disk spectrum and resulting emission-line intensities, we have computed modified models with AGNSPEC in which the standard $T_{\text {eff }}$ inside a break radius $R_{b}$ is multiplied by a factor $\left(R / R_{b}\right)^{1 / 4}$, following the discussion above. For the value of the break radius, we took $R_{b} / r_{g}=x_{b}\left(L / L_{\mathrm{Ed}}\right)$. We present results for $x_{b}=7$, which was motivated by a preliminary analysis but which serves here simply as an illustrative example. We used the same values of $M_{\mathrm{BH}}$ and $\dot{M}$ as used in our Series 1 and Series 2 models above. The run of $T_{\text {eff }}$ with radius for the modified models is shown in Figure 7. As expected, there is little effect for Series $1\left(f_{L}=9\right)$; but substantial alterations are seen for Series 2. In Figure 2, the Series 2 prediction for the $\mathrm{H} \beta$ equivalent width levels off above $\log T_{\max }=5.4$. This reduces but does not eliminate the discrepancy with the observed trend. Figure 3 shows a similar leveling of the predicted He II intensity. Figure 5 shows a substantial reduction in the predicted $\mathrm{Ne} \mathrm{V}$ intensity for $\log T_{\max }$ around 5.4. These models are not intended to achieve a detailed fit to the observed spectra, but they do illustrate the substantial effect on the ionizing continuum that results from a modified disk structure resulting from superEddington accretion.

\section{RADIO VERSUS NON-RADIO SOURCES}

Our focus has been on the entire SDSS quasar data set, which is strongly dominated by radio-quiet objects. However, radio loudness raises interesting questions regarding the black hole spin, which affects the inner disk boundary and thus the 


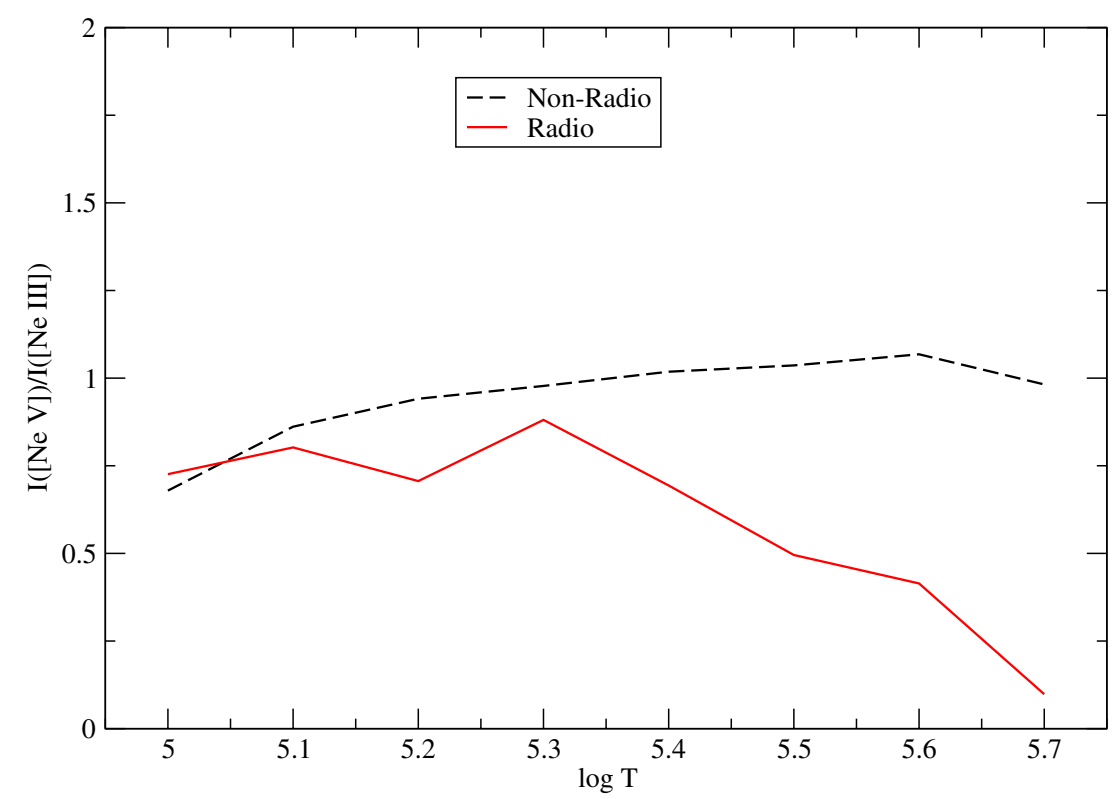

Figure 8. Comparison of the $[\mathrm{Ne} \mathrm{v}] /\left[\mathrm{Ne}\right.$ III] ratio for radio-detected and non-detected SDSS quasars as a function of the reference temperature $T_{\text {ref }}$. Note the significant difference for the higher values of $T_{\text {ref. }}$ See the text for discussion.

(A color version of this figure is available in the online journal.)

maximum temperature reached in the disk. Numerous authors have discussed the possibility that radio-loud AGN have rapidly spinning black holes (e.g., Blandford 1990), and on the other hand Garofalo et al. (2010) have proposed that radio-loud objects have retrograde black hole spin with respect to the disk angular momentum. The innermost stable circular orbit (ISCO) decreases from $6 R_{g}$ for $a=0$ to $1.22 R_{g}$ for $a_{*}=0.998$. Correspondingly, the radius of maximum effective temperature moves inward from $9.5 R_{g}$ to $1.55 R_{g}$; and the value of $T_{\max }$ increases from $10^{4.79} \dot{M}_{0}^{1 / 4} M_{8}^{-1 / 4}$ to $10^{5.43} \dot{M}_{0}^{1 / 4} M_{8}^{-1 / 4}$ (Shields 1989). For retrograde spin, the ISCO moves still farther out. These differences in the inner disk temperature could have a significant effect on the ionizing spectrum of the disk, while having less effect on the optical luminosity. Thus, we might expect differences between radio-loud and -quiet AGN in the observational quantities sensitive to $T_{\max }$ discussed above.

We have formed composite spectra separately for the radioloud and radio-quiet AGN, adhering to our previously defined bins in $T_{\text {ref. }}$. Here radio-loud was taken simply to mean detected in the Faint Images of the Radio Sky at Twenty Centimeters (FIRST) radio survey (Becker et al. 1995). This is not equivalent to the standard definition (Kellermann et al. 1989) and is distance-dependent, but it is sufficient to look for any qualitative difference between radio-loud and -quiet AGN. Figure 8 shows the observed trend of the [Ne v] intensity with $T_{\text {ref }}$ for the radioloud and -quiet composites. The [Ne v]/[Ne III] ratio is similar for the two groups for $\log T_{\text {ref }}=5.0$, but there is a decline for the radio-detected objects with increasing $T_{\text {ref }}$. Certainly, there is no evidence that $[\mathrm{Ne} \mathrm{V}]$ is stronger for the radio-detected objects, as might be expected if they have higher spin of a prograde sense. On the other hand, the weaker [ $\mathrm{Ne} \mathrm{v}$ ] of the radio objects might be expected if they involve retrograde disks.

\section{CONCLUSION}

The results presented here give focus to concerns about the disk model for the AGN continuum that have been voiced in the literature by numerous authors (see Section 1). In general terms, AGN over a wide range of $M_{\mathrm{BH}}$ and luminosity have rather similar SEDs (see Laor \& Davis 2011 and references therein). Organization of the observational data in terms of $T_{\text {ref }}$ helps to clarify what trends are expected on the basis of standard disk theory. We find that the expected trends in the intensity of $\mathrm{H} \beta$, He II, and [ $\mathrm{Ne} \mathrm{v}]$ are not seen in the observations. Over a range of a factor of five in the value of $T_{\text {ref }}$, the predicted disk temperature based on $M_{\mathrm{BH}}$ and $v L_{v}(5100)$, the [Ne v] intensity shows no systematic variation, and the $\mathrm{H} \beta$ equivalent width shows a reverse trend. While our models are highly simplified, in particular for cases that approach or exceed the Eddington limit, the basic immunity of these key line ratios as well as $\alpha_{\mathrm{os}}$ to the value of $T_{\text {ref }}$, which is essentially $1 / \operatorname{FWHM}(\mathrm{H} \beta)$, is a striking empirical fact. These results reinforce earlier studies indicating that a serious re-examination of the structure and emission mechanisms of the AGN central engine is in order. This might reasonably start with more detailed studies of the nature of disks in systems with high Eddington ratios, taking account of advection, winds, and all contributing sources of opacity.

We thank the anonymous referee for a careful and thorough review that greatly improved the final manuscript. We thank Ivan Hubeny for the use of the AGNSPEC program, R. Antonucci, O. Blaes, A. Laor, and B. Wills for helpful discussions. Funding for the Sloan Digital Sky Survey (SDSS) has been provided by the Alfred P. Sloan Foundation, the Participating Institutions, the National Aeronautics and Space Administration, the National Science Foundation, the U.S. Department of Energy, the Japanese Monbukagakusho, and the Max Planck Society. The SDSS Web site is http://www.sdss.org/. The SDSS is managed by the Astrophysical Research Consortium (ARC) for the Participating Institutions. The Participating Institutions are The University of Chicago, Fermilab, the Institute for Advanced Study, the Japan Participation Group, The Johns Hopkins University, the Korean Scientist Group, Los Alamos National Laboratory, the Max-Planck-Institute for Astronomy (MPIA), the Max-Planck-Institute for Astrophysics (MPA), New Mexico State University, University of Pittsburgh, University 
of Portsmouth, Princeton University, the United States Naval Observatory, and the University of Washington.

\section{REFERENCES}

Antonucci, R. R. J., \& Miller, J. S. 1985, ApJ, 297, 621

Becker, R. H., White, R. L., \& Helfand, D. J. 1995, ApJ, 450, 559

Begelman, M. C., King, A. R., \& Pringle, J. E. 2006, MNRAS, 370, 399

Bentz, M. C., Peterson, B. M., Netzer, H., Pogge, R. W., \& Vestergaard, M. 2009, ApJ, 697, 160

Blandford, R. D. 1990, in Active Galactic Nuclei, ed. T. J.-L. Courvoisser \& M. Mayor (Berlin: Springer), 161

Bonning, E. W., Cheng, L., Shields, G. A., Salviander, S., \& Gebhardt, K. 2007, ApJ, 659, 211

Boroson, T. A., \& Green, R. F. 1992, ApJS, 80, 109

Czerny, B., \& Elivis, M. 1987, ApJ, 321, 305

Czerny, B., Hryniewicz, K., Nikolajuk, M., \& Sadowski, A. 2011, MNRAS, 415, 2941

Deroches, L.-B., Greene, J. E., \& Ho, L. C. 2009, ApJ, 698, 1515

Ferland, G. J., Hu, C., Wang, J.-M., et al. 2009, ApJL, 707, L82

Ferland, G. J., Korista, K. T., Verner, D. A., et al. 1998, PASP, 110, 761

Garofalo, D., Evans, D. A., \& Sambruna, R. M. 2010, MNRAS, 406, 975

Hubeny, I., Blaes, O., Krolik, J. H., \& Agol, E. 2001, ApJ, 559, 680

Jiménez-Vicente, J., Mediavilla, E., Muñoz, J. A., \& Kochanek, C. S. 2012, ApJ, 751,106

Kaspi, S., Smith, P. S., Netzer, H., et al. 2000, ApJ, 533, 631

Kellermann, K. I., Sramek, R., Schmidt, M., Shaffer, D. B., \& Green, R. F. 1989, AJ, 98, 1195
Koratkar, A., \& Blaes, O. 1999, PASP, 111, 1

Korista, K., Ferland, G., \& Baldwin, J. 1997, ApJ, 487, 555

Laor, A., \& Davis, S. 2011, arXiv:1110.0653

Laor, A., Fiore, F., Elvis, M., Wilkes, B. J., \& McDowell, J. C. 1997, ApJ, 477,93

Lawrence, A. 2012, MNRAS, 423, 451

Malkan, M. 1983, ApJ, 268, 582

Morgan, C. W., Kochanek, C. S., Morgan, N. D., \& Falco, E. E. 2010, ApJ, 712,1129

Netzer, H. 1985, ApJ, 289, 451

Novikov, I. E., \& Thorne, K. S. 1973, in Black Holes, Les Houches, ed. C. DeWitt \& B. DeWitt (New York: Gordon \& Breach)

Osterbrock, D. E., \& Ferland, G. J. (ed.) 2006, Astrophysics of Gaseous Nebulae and Active Galactic Nuclei (2nd ed; Sausalito, CA: University Science Books), 343

Pooley, D., Blackburne, J. A., Rappaport, S., \& Schechter, P. L. 2007, ApJ, 661,19

Poutanen, J., Lipunova, G., Fabrika, S., Butkevich, A. G., \& Abolmasov, P. 2007, MNRAS, 377, 1187

Richards, G. T., Lacy, M., Storrie-Lombardi, L. J., et al. 2006, ApJS, 166, 470

Salviander, S., Shields, G. A., Gebhardt, K., \& Bonning, E. W. 2007, ApJ, 662,131

Shakura, N. I., \& Sunyaev, R. A. 1973, A\&A, 24, 337

Shang, Z., Brotherton, M. S., Green, R. F., et al. 2005, ApJ, 619, 41

Shields, G. A. 1978, Natur, 272, 706

Shields, G. A. 1989, NYASA, 571, 110

Shields, G. A., Gebhardt, K., Salviander, S., et al. 2003, ApJ, 583, 124

Thorne, K. S. 1974, ApJ, 191, 507 